\title{
Operation lifetimes of organic light-emitting devices with different layer structures
}

\author{
Jiun-Haw Lee ${ }^{\mathrm{a}, *}$, JianJang Huang ${ }^{\mathrm{a}}$, Chi-Chih Liao ${ }^{\mathrm{b}}$, Pier-Jy Hu ${ }^{\mathrm{b}}$, Yih Chang ${ }^{\mathrm{b}}$ \\ a Department of Electrical Engineering, Graduate Institute of Electro-Optical Engineering, National Taiwan University, No. 1, Sec. 4, \\ Roosevelt Road, Taipei, Taiwan

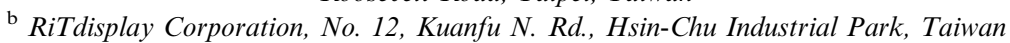

Received 24 June 2004; in final form 26 November 2004

Available online 5 January 2005

\begin{abstract}
In this Letter, we present and analyze the operation lifetime behaviors of organic light-emitting devices by changing thicknesses of the hole-transport layer (HTL), the emitting layer (EML) and the electron transport layer (ETL). It is found that devices with higher power efficiency exhibit longer lifetimes. Given the same power efficiency, devices with a thicker HTL (or a thinner EML and ETL) have longer lifetimes. We also observe that, among different samples, the fixed charge density increasing rate near the HTL/EML interface is linearly correlated to the non-radiative center formation rate.
\end{abstract}

(C) 2004 Elsevier B.V. All rights reserved.

\section{Introduction}

The organic light-emitting device (OLED) is one of the most promising candidates for the next generation display technologies since it exhibits advantages of low-power consumption, high brightness, high contrast, wide-view angle, and potentially low cost $[1,2]$. However, the operation lifetime is still one of the major limitations for OLED applications as compared to other competitive display technologies such as semiconductor light-emitting diodes (LEDs) and liquid crystal displays (LCDs).

During the long-time operation of an OLED, indium ions from the indium-tin-oxide (ITO) anode and metal ions from the cathode $(\mathrm{Mg}: \mathrm{Ag}$ or $\mathrm{LiF} / \mathrm{Al})$ will diffuse through the hole transport layer (HTL) and the electron transport layer (ETL) into the emitting layer (EML) $[3,4]$. They form fluorescence quenchers and decrease

\footnotetext{
${ }^{*}$ Corresponding author. Fax: +886 223677467.

E-mail address: jhlee@cc.ee.ntu.edu.tw (J.-H. Lee).
}

the luminance under a fixed driving current. Meanwhile, the mobile ions also create a built-in voltage that increases the driving voltage under the same electrical current. On the other hand, the unstable cation in the EML is another degradation mechanism proposed in [5]. Since the HTL/EML hetero-junction in an OLED confines the carriers, they pile up near the interface when applying an electrical field. Injected holes from the HTL to the EML form cations in the EML material which are chemically unstable [6]. It will accelerate the formation of non-radiative trapping centers and result in luminance decay and voltage increase of a device. Kondakov et al. found that the decrease of luminance efficiency is linearly correlated to the accumulation of immobile positive charges at the HTL/EML interface. The electrical aging will generate carrier traps which act as non-radiative centers [7].

In this Letter, we conduct a series of experiments with the same organic materials but with different device structures. Devices with thicker HTLs or ETLs do not necessarily processes longer lifetimes. That is, metal ion diffusion is not the major lifetime mechanism in 
our experiment. On the contrast, the operation lifetime decreases with the increase of layer thicknesses. We found that devices with higher power efficiency (in terms of $1 \mathrm{~m} / \mathrm{W}$ ) have longer device lifetimes. For devices with the same power efficiency, those with more holes at EML have shorter operation lifetimes. That implies that the operation lifetimes are effected by the cation formation in the EML. Using the model developed by Kondakov et al., it was shown that the fixed charge formation rate is proportional to that of quencher formation at the HTL/EML interface in our experiment. Although, it is not the direct evidence to prove that the generated trapping carriers become non-radiative centers during electrical aging, it shows, to some degree, those two parameters correlated to each other. We will present experimental results and related discussions in Section 2 and conclude in Section 3.

\section{Results and discussion}

In all of our experiments, we used ITO glass substrates with low sheet resistivity $(10 \mathrm{Ohm} / \mathrm{square})$ and flat surface roughness $(\mathrm{Ra}<1 \mathrm{~nm})$. The size of the active region in our test pixel is $1 \mathrm{~cm} \times 1 \mathrm{~cm}$. In our devices, we used copper-phthalocyanine $(\mathrm{CuPc})$ as the hole injection layer (HIL) material, $N, N^{\prime}$-diphenyl- $N, N^{\prime}$-bis(1napthyl)-1,1'-biphenyl-4,4'-diamine (NPB) as the HTL material, 9-benzothiazol-2-yl-1,1,6,6-tetramethyl-2,3,5, 6-tetrahydro-1H,4H-11-oxa-3a-aza-benzo anthracene10 -one (C545T) as the green dopant of the EML, tris(8-hydroxyquinoline) aluminum (Alq3) as the ETL material and the host material of the EML. The doping concentration of C545T in Alq3 was 1.33\% in mole ratio. $\mathrm{LiF}$ of $1.2 \mathrm{~nm}$ in thickness was used for more efficient electron injection between the ETL and the aluminum cathode. The thickness of the aluminum cathode was $100 \mathrm{~nm}$. After organic and metal deposition, we encapsulated the devices in the glove box with $\mathrm{O}_{2}$ and $\mathrm{H}_{2} \mathrm{O}$ concentrations below $1 \mathrm{ppm}$. Prior to the lifetime test, devices were characterized with a Keithley 2400 source meter for current-voltage measurement and with a SR-2 photometer for the brightness measurement. In our lifetime test, we fixed the initial luminance at 1000 $\mathrm{cd} / \mathrm{m}^{2}$ and supplied a constant current to our devices. The brightness and voltage were recorded with a photodiode and a Keithley 2400 source meter at different durations.

Table 1 illustrates the device structures and the device performance. The current efficiency and power efficiency are deduced from the initial voltage and the driving current at the brightness of $1000 \mathrm{~cd} / \mathrm{m}^{2}$. The lifetime value used here is half-lifetime, i.e., the time required to obtain half initial brightness under the constant current mode. In our experiments, the HIL thickness is fixed at $25 \mathrm{~nm}$ and we vary the HTL, EML and ETL thicknesses.
Device 1 is the basic structure. The thickness of the HIL, HTL, EML and ETL is 25, 35, 27.5, and 27.5 $\mathrm{nm}$, respectively.

As shown in Table 1, the HTL and ETL thickness is varied from 15 to $75 \mathrm{~nm}$ in device 1, 2, 3 and 4, and 27.5 to $67.5 \mathrm{~nm}$ in device 1,5 and 6 . To achieve better optical interference effect and less metal-quench effect from the cathode, the thickness of the ETL is always kept larger than $27.5 \mathrm{~nm}$. The EML thickness varies from 10 to $27.5 \mathrm{~nm}$, while the total thickness of the EML and the ETL is kept constant in order to provide the same optical interference path in device $1,7,8$, and 9. In a conventional NPB/Alq3 device, the difference of the highest occupied molecular orbital between these two materials $(0.3 \mathrm{eV})$ is less than the lowest unoccupied molecular orbital difference $(0.6 \mathrm{eV})$. It is usually assumed holes penetrate from NPB into Alq3 and recombine with electrons. In Alq3, the hole mobility is one order of magnitude smaller than the electron mobility [8]. Therefore, the recombination zone ranges from the EML to the HTL/EML interface. Excitons formed in this region are likely to diffuse and emit light. The exciton diffusion length is estimated to be about $20 \mathrm{~nm}$ [2]. The emitted light inside an OLED experiences two major reflections, i.e., on the ETL/cathode and glass/ITO interfaces. The current efficiency (in terms of cd/A), which benchmarks the conversion of electrical carriers to optical photons radiated out of a device, has only a $2.5 \%$ difference with various HTL thicknesses in device 1, 3 and 4. Device 2 has an extremely low current efficiency which will be explained later. Furthermore, current efficiencies decrease with increasing (EML + ETL) thicknesses when comparing device 1, 5 and 6 in Table 1 . Therefore, it is clear that the optical interference effect does not play a major role in determining the current efficiency.

As shown in Table 1, the operation lifetime of device 1 is $1061 \mathrm{~h}$. Assuming the lifetime is inversely proportional to the luminance [9], we can obtain a $10000 \mathrm{~h}$ lifetime when devices are operated at $100 \mathrm{~cd} / \mathrm{m}^{2}$. As mentioned above, the current efficiency of all devices exceeds $13 \mathrm{~cd} / \mathrm{A}$ and is within $5 \%$ difference except in device 2 and 9. Device 2 has the thinnest HTL (15 $\mathrm{nm})$. We can see that the driving current is much higher than other devices. Since the roughness of the HIL material (CuPC) is much larger than other organic materials, HTL needs to be thicker than $20 \mathrm{~nm}$ to cover the surface for lower leakage currents. While in device 9 , the thinnest EML, doped with high IQE material, leads to an incomplete energy transfer and a low current efficiency. To verify the process stability, devices 1,5 and 7 were fabricated repeatedly one year after the original devices have been made. Device performance is also illustrated in Table 1 and marked by a star signal. Although, devices with the same structures were fabricated at different time, similar results with the tolerance less than $10 \%$ can be obtained. However, in the discussions below 
Table 1

Device structure and the corresponding performance with initial luminance at $1000 \mathrm{~cd} / \mathrm{m}^{2}$ under constant driving currents

\begin{tabular}{|c|c|c|c|c|c|c|c|c|c|c|}
\hline No. & $\begin{array}{l}\text { HTL } \\
\text { thickness } \\
(\mathrm{nm})\end{array}$ & $\begin{array}{l}\text { EML } \\
\text { thickness } \\
(\mathrm{nm})\end{array}$ & $\begin{array}{l}\text { ETL } \\
\text { thickness } \\
(\mathrm{nm})\end{array}$ & $\begin{array}{l}\text { EML + ETL } \\
\text { thickness (nm) }\end{array}$ & Lifetime (h) & $\begin{array}{l}\text { Initial } \\
\text { voltage }(\mathrm{V})\end{array}$ & $\begin{array}{l}\text { Driving } \\
\text { current } \\
(\mathrm{mA})\end{array}$ & $\mathrm{cd} / \mathrm{A}$ & $\mathrm{lm} / \mathrm{W}$ & Remark \\
\hline 1 & 35 & 27.5 & 27.5 & 5 & $\begin{array}{l}1061 \\
1121^{*}\end{array}$ & $\begin{array}{l}5.137 \\
4.992 *\end{array}$ & $\begin{array}{l}6.6 \\
7 *\end{array}$ & $\begin{array}{l}15.2 \\
14.3^{*}\end{array}$ & $\begin{array}{l}9.27 \\
8.99 *\end{array}$ & $\mathrm{~h}$ \\
\hline 2 & 15 & 27.5 & 27.5 & 55 & 685 & 4.679 & 15 & 6.7 & 4.48 & $\mathrm{~h}$ \\
\hline 3 & 55 & 27.5 & 27.5 & 55 & 987 & 5.908 & 6.4 & 15.6 & 8.31 & $\mathrm{e}$ \\
\hline 4 & 75 & 27.5 & 27.5 & 55 & 920 & 6.765 & 6.5 & 15.4 & 7.14 & $\mathrm{e}$ \\
\hline 5 & 35 & 27.5 & 47.5 & 75 & $\begin{array}{l}844 \\
808^{*}\end{array}$ & $\begin{array}{l}5.833 \\
5.773 *\end{array}$ & $\begin{array}{l}6.8 \\
6.2^{*}\end{array}$ & $\begin{array}{l}14.7 \\
16.1^{*}\end{array}$ & $\begin{array}{l}7.92 \\
8.78^{*}\end{array}$ & $\mathrm{~h}$ \\
\hline 6 & 35 & 27.5 & 67.5 & 95 & 735 & 6.738 & 7.5 & 13.3 & 6.22 & $\mathrm{~h}$ \\
\hline 7 & 35 & 20 & 35 & 55 & $\begin{array}{l}1112 \\
1225^{*}\end{array}$ & $\begin{array}{l}5.056 \\
5.358^{*}\end{array}$ & $\begin{array}{l}6.4 \\
6^{*}\end{array}$ & $\begin{array}{l}15.6 \\
16.7^{*}\end{array}$ & $\begin{array}{l}9.71 \\
9.77 *\end{array}$ & $\mathrm{~b}$ \\
\hline 8 & 35 & 15 & 40 & 55 & 1129 & 5.062 & 6.4 & 15.6 & 9.7 & $\mathrm{~b}$ \\
\hline 9 & 35 & 10 & 45 & 55 & 901 & 4.41 & 16 & 6.3 & 4.45 & $\mathrm{e}$ \\
\hline
\end{tabular}

* Device performances of OLEDs those were fabricated one year after the others have been made.

we still use the original data (data without the star signal) for comparisons.

Fig. 1 shows the device lifetime and the power efficiency versus the layer thickness. In our experiment, the power efficiency was measured at the brightness of 1000 $\mathrm{cd} / \mathrm{m}^{2}$. In this figure, solid curves with solid symbols show the device lifetime and dashed curves with open symbols show the power efficiency. The device lifetime and the power efficiency among these three pairs of curves follow the same trend. With a thicker HTL, the power efficiency drops since the driving voltage is higher. On the other hand, with a thinner HTL, the power efficiency decreases because the current efficiency is lower (see device 2). When varying the thickness of the EML, the power efficiency achieves a maximum at $15 \mathrm{~nm}$.

It was previously proposed that mobile ions were the dominant factor in the OLED degradation. Devices with thicker ETLs would have longer lifetime because the effect of metal ions from the cathode was smaller.

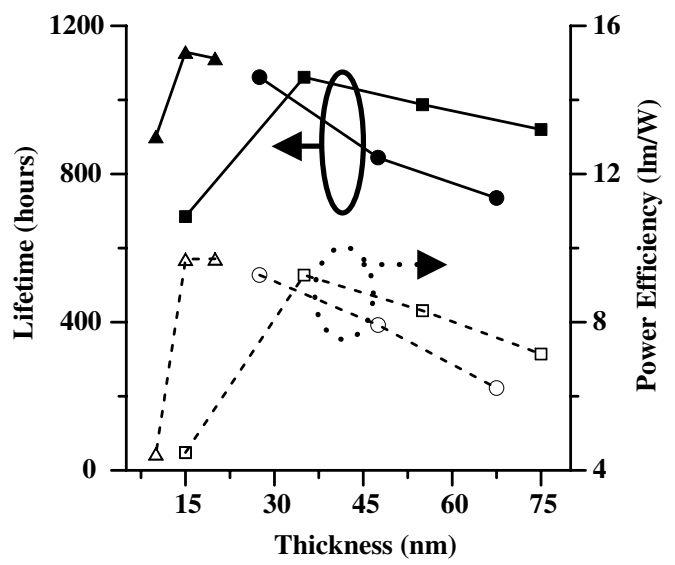

Fig. 1. Dependence of power efficiencies and device lifetimes on different organic layer thicknesses. Solid lines and symbols correspond to lifetimes while dash line and open symbols corresponds to power efficiencies. Square, triangular and round symbols represent different HTL, EML and ETL thicknesses.
Likewise, oxygen atoms from the anode will attack the device more seriously when the HTL becomes thinner. However, our experiments demonstrated different results. As shown in Fig. 1, when the thickness of the HTL and ETL increases, the operation lifetime first increases and then decreases. The lifetime trend is quite similar to that of the power efficiency. That means the mobile ion theory about the anode and the cathode does not apply in our experiments. And it shows a correlation between the power efficiency and the operating lifetime.

As shown in Table 1, devices 7 and 8 show the highest power efficiency (around $9.7 \mathrm{~lm} / \mathrm{W}$ ) and the longest lifetime (1112 and $1129 \mathrm{~h}$ ) among all of the devices. These two structures have been optimized electrically and optically to achieve the charge-balance condition. Since the dopant plays a role as a trap, the driving voltage decreases in device 9 in a smaller doped region. When the total thickness of EML and ETL is kept constant, a device with a thinner EML thickness demonstrates higher electron mobility due to fewer traps. In this case, electrons and holes are not balanced in the HTL/EML interface. Since the device 9 has higher equivalent electron mobility than that of the device 7 and 8, electrons outnumber holes near the HTL/EML interface during electron-capture process. Thus excess electrons will accumulate near this interface and then be blocked by the potential barrier of NPB in device 9. We define it as an 'electron-rich' device. Similarly, the EML thickness of device 1 is larger than that of devices 7 and 8 while the (EML + ETL) thickness is kept the same. Hence, it is a 'hole-rich' device. With such kind of methodology, we can define those experiments to electronrich, hole-rich and balanced devices as shown in the last column of Table 1.

Typically, a higher power efficiency device has a longer device lifetime and thus the generated heat accounts for the device degradation. Because of the low energy efficiency of OLEDs (typically less than 10\%), 
the generated heat during operation is one of the main issues that cause organic material degradation. It was discussed by Sturm et al. [10].

Fig. 2 shows the dependence of the lifetime on the power efficiency for both electron-rich and hole-rich devices. The two curves in Fig. 2 coincide at the highest efficiency and the highest lifetime since, at his point, it reaches charge balance condition and splits at lower efficiency. For electron-rich devices, their lifetimes drop and saturate at about $900 \mathrm{~h}$ along with the decreasing power efficiency. However, for the hole-rich devices, lifetime is always shorter than electron-rich devices under the same power efficiency. In hole-rich devices, some of the holes at the EML form unstable Alq3 cations and hence decrease the lifetime. For electron-rich devices, holes penetrated into the EML but recombine very quickly since the hole density is much less than the electron density. Hence, the lifetime in electron-rich condition seems to achieve saturation even the power efficiency is quite low.

As shown in [7], during the operation of an OLED, fixed charges will be generated near the HTL/EML interface and hinder further charge injection and transportation. Hence, to sustain the same electrical current, a higher voltage is required. The relationship between driving voltage increase and fixed charge density can by represented as follows according to the model proposed by [7]:

$V_{0} \propto \frac{Q_{0}}{C_{(\mathrm{EML}+\mathrm{ETL})}}$,

where $V_{0}$ is the voltage increased by the interface fixed charge, $Q_{0}$ is the fixed charge at the HTL/EML interface, $\left.C_{(\mathrm{EML}}+\mathrm{ETL}\right)$ is the capacitance of EML and ETL layers in series. Therefore, the corresponding charge density (i.e., $Q_{0}$ divided by the area of the device) can be represented as



Fig. 2. Dependence of lifetimes on power efficiencies. Dash line and square symbol correspond to electron-rich devices. Solid line and triangular symbol represent hole-rich devices.
$V_{0} \propto \frac{\sigma_{0}}{\varepsilon_{0} \varepsilon_{\mathrm{r}}} d_{(\mathrm{EML}+\mathrm{ETL})}$.

Here, $\varepsilon_{\mathrm{r}}$ is the relative dielectric constant of the $(\mathrm{EML}+\mathrm{ETL}), \varepsilon_{0}$ is the permittivity of free space and $\left.d_{(\mathrm{EML}}+\mathrm{ETL}\right)$ is the thickness of the (EML+ETL). By differentiating both sides, we have

$\frac{\partial}{\partial t} V_{0} \propto d_{(\mathrm{EML}+\mathrm{ETL})} \frac{\partial}{\partial t} n_{\mathrm{e}} \propto \frac{\partial}{\partial t} V$,

where $n_{\mathrm{e}}$ is the charge density and $V$ is the driving voltage. If the charge density increase rate is proportional to photon density decrease rate, it can be represented as

$\frac{\partial}{\partial t} V \propto d_{(\mathrm{EML}+\mathrm{ETL})} \frac{\partial}{\partial t} n_{\mathrm{ph}}$,

where $n_{\mathrm{ph}}$ is the photon density. The typical luminance decay and voltage increase curves are shown in the inset of Fig. 3. After a fast luminance decay in the first $40 \mathrm{~h}$, the curves then decrease linearly. The lifetime at $50 \%$ initial luminance in Table 1 is extrapolated from 80 to 170 $\mathrm{h}$. The corresponding voltage increase rate, $\mathrm{mV} / \mathrm{h}$, and luminance decay rate, $\% / \mathrm{h}$, are also obtained from those curves. They behave linearly with time. As shown in Fig. 3 , the product of the photon density decrease rate and the total thickness of EML and ETL versus voltage increase rate are linearly correlated to each other on all 9 devices. There appears to be a direct relationship between the voltage increase and photon decrease. However, the $15 \% / \mathrm{h} * \AA$ offset luminance decay rate at 0 voltage rise implies there should be an offset hidden in Eqs. (1)-(4). In this case, since the energy transfer distance is limited, we believe a photon density change occurs during charge density accumulation. However, it is out of the scope of this Letter.



Fig. 3. Voltage increase rate versus the term 'luminance decay rate times the total thickness of EML and ETL'. The inset shows typical curves of luminance decay and voltage as a function of time. 


\section{Conclusions}

In summary, we have discussed device performance and operation lifetimes with different device structures. Devices with higher power efficiencies exhibit longer lifetimes. A thicker device does not have a longer lifetime. It implies the mobile-ion diffusion from the electrode is not the dominant factor in our experiments. Under chargeunbalanced conditions, a hole-rich device had a shorter lifetime than an electron-rich device under the same power efficiency. Also, the fixed charge increase rate and non-radiative recombination center formation rate were linearly correlated to each other.

\section{Acknowledgements}

This work is supported by the AIXTRON Taiwan Corporation.

\section{References}

[1] C.W. Tang, S.A. Vanslyke, Appl. Phys. Lett. 51 (1987) 913

[2] C.W. Tang, S.A. Vanslyke, C.H. Chen, J. Appl. Phys. 65 (1989) 3610.

[3] J. Shen, D. Wang, E. Langlois, W.A. Barrow, P.J. Green, C.W. Tang, J. Shi, Synth. Met. 111-112 (2000) 233.

[4] S.T. Lee, Z.Q. Gao, L.S. Hung, Appl. Phys. Lett. 75 (1999) 1108.

[5] G. Vamvounis, H. Aziz, N.X. Hu, Z.D. Popovic, Synth. Met. 143 (2004) 69.

[6] Z.D. Popovic, H. Aziz, IEEE J. Sel. Top. Quantum Electron. 8 (2002) 362.

[7] D.Y. Kondakov, J.R. Sandifer, C.W. Tang, R.H. Young, J. Appl. Phys. 93 (2003) 1108

[8] W. Brütting, S. Berleb, A.G. Mückl, Org. Electron. 2 (2001) 1.

[9] S.A. Van Slyke, C.H. Chen, C.W. Tang, Appl. Phys. Lett. 69 (1996) 2160.

[10] J.C. Sturm, W. Wilson, M. Iodice, IEEE J. Sel. Top. Quantum Electron. 4 (1998) 75. 